\title{
The upregulated expression of vascular endothelial growth factor in surgically treated patients with recurrent/radioresistant cervical cancer of the uterus
}

\author{
KOSUKE YOSHIDA $^{1}$, SHIRO SUZUKI $^{1}$, JUN SAKATA $^{1}$, FUMI UTSUMI $^{1}$, KAORU NIIMI $^{1}$, \\ NOBUHISA YOSHIKAWA ${ }^{1}$, KIMIHIRO NISHINO ${ }^{1}$, KIYOSUMI SHIBATA ${ }^{2}$, \\ FUMITAKA KIKKAWA $^{1}$ and HIROAKI KAJIYAMA ${ }^{1}$
}

\author{
${ }^{1}$ Department of Obstetrics and Gynecology, Graduate School of Medicine, Nagoya University, Showa-ku, Nagoya 466-8550; \\ ${ }^{2}$ Department of Obstetrics and Gynecology, Banbuntane Hotokukai Hospital, Nakagawa-ku, Nagoya 454-8509, Japan
}

Received May 25, 2017; Accepted September 20, 2017

DOI: $10.3892 / \mathrm{ol} .2018 .8610$

\begin{abstract}
Vascular endothelial growth factor (VEGF) inhibitors have been utilized for the treatment against advanced or recurrent cervical carcinoma as a novel therapeutic modality. However, the expression level of VEGF in post-radiotherapy relapsed/persistent cervical cancer remains to be elucidated. The aim of the present study was to investigate the expression of VEGF and associated molecules using tumor samples from patients with post-radiotherapy relapsed/persistent cervical cancer. From a database of 826 patients who were treated at our institution between 2003 and 2015, eight patients with post-radiotherapy relapsed/persistent cervical cancer were identified, and 20 patients who underwent initial surgery alone were used as a control. Using samples from these patients, the expression levels of VEGF-A, VEGF receptor-1 (VEGFR-1) and hypoxia inducible factor- $1 \alpha$ (HIF-1 $\alpha$ ) were immunohistochemically categorized as negative or weakly, moderately, or strongly positive according to the size of the staining area, and intensity. In carcinoma cells, the expression levels of VEGF-A, VEGFR-1 and HIF-1 $\alpha$ were significantly higher in post-radiotherapy relapsed/persistent cervical cancer compared with control patients ( $\mathrm{P}=0.0003,0.0003$, and 0.0001 , respectively). In stroma cells, similar tendencies with statistical significance were observed $(\mathrm{P}=0.0014$ and $\mathrm{P}<0.0001$, respectively). In addition, the expression levels of VEGF-A and VEGFR-1 in carcinoma cells were significantly correlated with each other
\end{abstract}

Correspondence to: Dr Hiroaki Kajiyama, Department of Obstetrics and Gynecology, Graduate School of Medicine, Nagoya University, Tsuruma-cho 65, Showa-ku, Nagoya 466-8550, Japan E-mail: kajiyama@med.nagoya-u.ac.jp

Abbreviation: PRRCC, post-radiotherapy relapsed/persistent cervical cancer

Key words: uterine cervical cancer, radioresistance, VEGF, VEGFR-1, HIF-1 $\alpha$
$(\mathrm{P}<0.0001)$. A significantly higher expression of VEGF was identified in post-radiotherapy relapsed/persistent cervical cancer compared with typical specimens from cervical cancer. The findings provide a novel insight into the clinical treatment for recurrent/persistent cervical cancer using a VEGF antagonist.

\section{Introduction}

Cervical cancer is one of the most common malignancies in females worldwide. Mortality rates associated with uterine cervical cancer have declined due to the widespread use of cancer screening for the prevention and early detection of cervical cancer (1). Moreover, since concurrent chemoradiotherapy (CCRT) has been established as a standard treatment, the prognosis of those patients has improved $(2,3)$. However, about one third of patients experience recurrence within five years (4), with a median survival after recurrence of 15 months (5), and less than $5 \%$ of them survive for 5 years (6). Thus, the oncologic outcome is far from satisfactory. Especially, the prognosis of patients with recurrent disease within a previously irradiated field is unfavorable (7). In addition, earlier studies indicated that response rates to chemotherapy in those patients were poorer than that of those with out-of-field recurrence $(8,9)$. Therefore, the oncologic outcome of patients with post-radiotherapy relapsed/persistent cervical cancer (PRRCC) is still poor. Recently, bevacizumab, a humanized monoclonal antibody targeting vascular endothelial growth factor A (VEGF-A), has been approved for this tumor, and moreover immunotherapy is under investigation (10).

VEGF-A is a multifunctional and an important molecule in endothelial signaling and angiogenesis. VEGF-A binds to its receptor VEGFR-1, and the downstream signaling is thought to be involved in cancer proliferation and invasion (11). There have been several reports showing that the overexpression of VEGF in cancer cells or serum is correlated with radioresistance and poor disease-free survival (12-16), and a meta-analysis suggested that high expressions of VEGF was significantly associated with poor survival outcome (17). Although VEGF inhibitors such as bevacizumab are widely used against several 
solid cancers, evidence regarding their efficacy against cervical cancer is not satisfactory, especially PRRCC. Particularly, to our best knowledge, there have been no reports on the expression level of VEGF in PRRCC based on the fact that surgical treatment is rarely performed as a salvage therapy for those patients (6). In sophisticated randomized clinical trials, the addition of a VEGF inhibitor such as bevacizumab to combination chemotherapy led to a significant improvement of the oncologic outcome of patients with recurrent, persistent, and highly metastatic cervical cancer $(18,19)$. Accordingly, bevacizumab has been applied in actual clinical practice for this tumor. These results led us to hypothesize that the expression of VEGF may be upregulated in those patients.

Reviewing 826 clinical records of cervical cancer patients in our institute from 2003 to 2015, we identified eight patients with PRRCC who underwent debulking surgery, and evaluated the expression of VEGF immunohistochemically. In the present study, we further clarify the upregulation of VEGF in radioresistant cervical cancer by evaluating the expressions of VEGFR-1 and hypoxia inducible factor-1 $\alpha$ (HIF-1 $\alpha)$.

\section{Materials and methods}

Patients. We retrospectively reviewed all the records of 826 patients with cervical cancer who were initially treated in our hospital from 2003 to 2015. Written informed consent was acquired from all patients. This study was approved by the Ethics Committee of our institute (Approval no. 2013-0078). Treatment strategies for each patient were determined by several gynecologic oncologists in our hospital depending on their age, performance status (PS), and spread of the disease. For example, as primary treatments, patients who were in the early stage and had a good PS were indicated for radical hysterectomy, and the other patients were treated with CCRT or radiotherapy alone. As treatments for recurrence, most patients were treated with chemotherapy, and only a few patients with localized disease were selected for surgical resection.

Ninety-seven patients had PRRCC, and 14 of them underwent surgery for a recurrent lesion. After excluding six patients because of lymph node metastasis or small residual tumors, eight patients with uterine or vaginal stump recurrence were investigated. Twenty patients who underwent radical surgery without neoadjuvant therapy were extracted as a control (Fig. 1).

Immunohistochemical (IHC) staining and its evaluation. Archival formalin-fixed, paraffin-embedded tumor tissue obtained at surgery was used in this study. Sections of $4-\mu \mathrm{m}$ thickness were prepared using a microtome. The sections were deparaffinized and rehydrated, subjected to antigen retrieval in $10 \mathrm{mM}$ sodium citrate ( $\mathrm{pH} 6.0$ ) for $20 \mathrm{~min}$ at $95^{\circ} \mathrm{C}$ in a microwave, and treated with $0.3 \%$ hydrogen peroxide in methanol for 20 min after being washed with phosphate-buffered saline (PBS). Then, the sections were blocked with appropriate serum using Histofine SAB-PO(R) kit or Histofine SAB-PO(M) kit according to the manufacturer's protocol (Nichirei, Tokyo, Japan), and incubated with an appropriate first antibody diluted by PBS at $4^{\circ} \mathrm{C}$ overnight. After rinsing with PBS, the sections were incubated with an appropriate second antibody, and then peroxidase labeled streptavidin using the kit. Then, the sections were rinsed with PBS and developed by the 3, 3'-diaminobenzidine (DAB) substrate-chromogen. After rinsing in water, the sections were incubated with hematoxylin, dehydrated, and mounted. Details about the reagents are presented in Table I.

Based on the IHC activity, a four-tiered semi-quantitative score was assigned according to the intensity and area of stained cells, as follows: For the evaluation of IHC expression, the staining intensity was scored as: 0 , negative; 1 , weak; 2 , medium; or 3 , strong. The percentage of the staining area was scored as 'focal' (1-10\%), 'sporadic' (11-50\%), and diffuse $(>51 \%)$ relative to the total tumor area. Carcinoma cells and stroma were separately evaluated by two researchers, and the final score was decided according to Table II ('negative', 'weak', 'moderate', and 'strong', respectively).

All photographs were taken using Zeiss Axio Imager.A1 (Carl Zeiss, Tokyo, Japan).

Statistics. All statistical analyses were performed with EZR (Saitama Medical Center, Jichi Medical University, Saitama, Japan), which is a graphical user interface for $\mathrm{R}$ (The $\mathrm{R}$ Foundation for Statistical Computing, Vienna, Austria). More precisely, it is a modified version of $\mathrm{R}$ commander designed to add statistical functions frequently used in biostatistics. Differences between recurrent cancer and control patients were assessed by the Mann-Whitney $U$ test and t-test, and the correlation of each expression was assessed by Spearman's correlation coefficient. Differences at $\mathrm{P}<0.05$ were considered significant.

\section{Results}

We first compared clinical backgrounds of eight patients with PRRCC and those of 20 patients with primary uterine cervical cancer. Distributions of the age, tumor size, and lymphovascular space invasion between the two groups were not significantly different. All patients had squamous cell carcinoma (SCC), and the serum SCC level at surgery was not significantly different (Table III). Detailed characteristics of PRRCC patients are presented in Table IV. All of the patients had previously received radiotherapy, and six of them had received CCRT. Salvage hysterectomy was performed for seven patients, and pelvic exenteration was performed for case 2 , who had vaginal stump recurrence after vaginal total hysterectomy for carcinoma in situ (CIS).

Representative images of IHC are shown in Fig. 2. In both carcinoma and stroma cells, the expressions of VEGF-A were significantly higher in the PRRCC group than in controls [PRRCC vs. control: $\mathrm{P}=0.0003$ (carcinoma) and $\mathrm{P}=0.0014$ (stroma), respectively] (Fig. 2A and Table VA). Similarly, the expressions of VEGFR-1 were also significantly stronger [PRRCC vs. control: $\mathrm{P}=0.0003$ (carcinoma) and $\mathrm{P}<0.0001$ (stroma), respectively] (Fig. $2 \mathrm{~B}$ and Table VB). Of note, the expressions of VEGF-A and VEGFR-1 in carcinoma cells were significantly correlated with each other (Spearman's correlation coefficient: $0.856 ; \mathrm{P}<0.0001$ ) (Fig. 3A). In addition, the correlation of the VEGF-A expression in carcinoma cells and the VEGFR-1 expression in stroma cells was moderate (Spearman's correlation coefficient: 0.484; $\mathrm{P}=0.0090$ ) (Fig. 3B), and that of the VEGF-A expression in stroma cells and the VEGFR-1 expression in carcinoma 
Table I. Details about antibodies and immunohistochemistry kits.

A, Primary antibodies

\begin{tabular}{|c|c|c|c|c|}
\hline Antibody name & Manufacturer & Product no. & Host & Dilution rate \\
\hline Anti-VEGF antibody & Abcam & ab46154 & Rabbit & $1: 100$ \\
\hline Anti-VEGF receptor 1 antibody & Abcam & $\mathrm{ab} 2350$ & Rabbit & $1: 100$ \\
\hline Anti-HIF- $1 \alpha$ antibody & Abcam & ab1Rabbit & $1: 100$ & \\
\hline
\end{tabular}

B, Immunohistochemistry kits

\begin{tabular}{lcc}
\hline Kit name & Manufacturer & Product no. \\
\hline Histofine SAB-PO (R) kit & Nichirei & 424032 \\
$10 \%$ Normal goat serum & \\
Biotin labeled anti-rabbit IgG antibody & \\
Peroxidase labeled streptavidin & Nichirei & 424022 \\
Histofine SAB-PO (M) kit & \\
$10 \%$ Normal rabbit serum & \\
$\begin{array}{l}\text { Biotin labeled anti-mouse IgG + IgA + IgM antibody } \\
\text { Peroxidase labeled streptavidin }\end{array}$ & \\
Liquid DAB+ Substrate Chromogen system & Dako & \\
\hline
\end{tabular}

VEGF, vascular endothelial growth factor; HIF-1 $\alpha$, hypoxia inducible factor- $1 \alpha$.

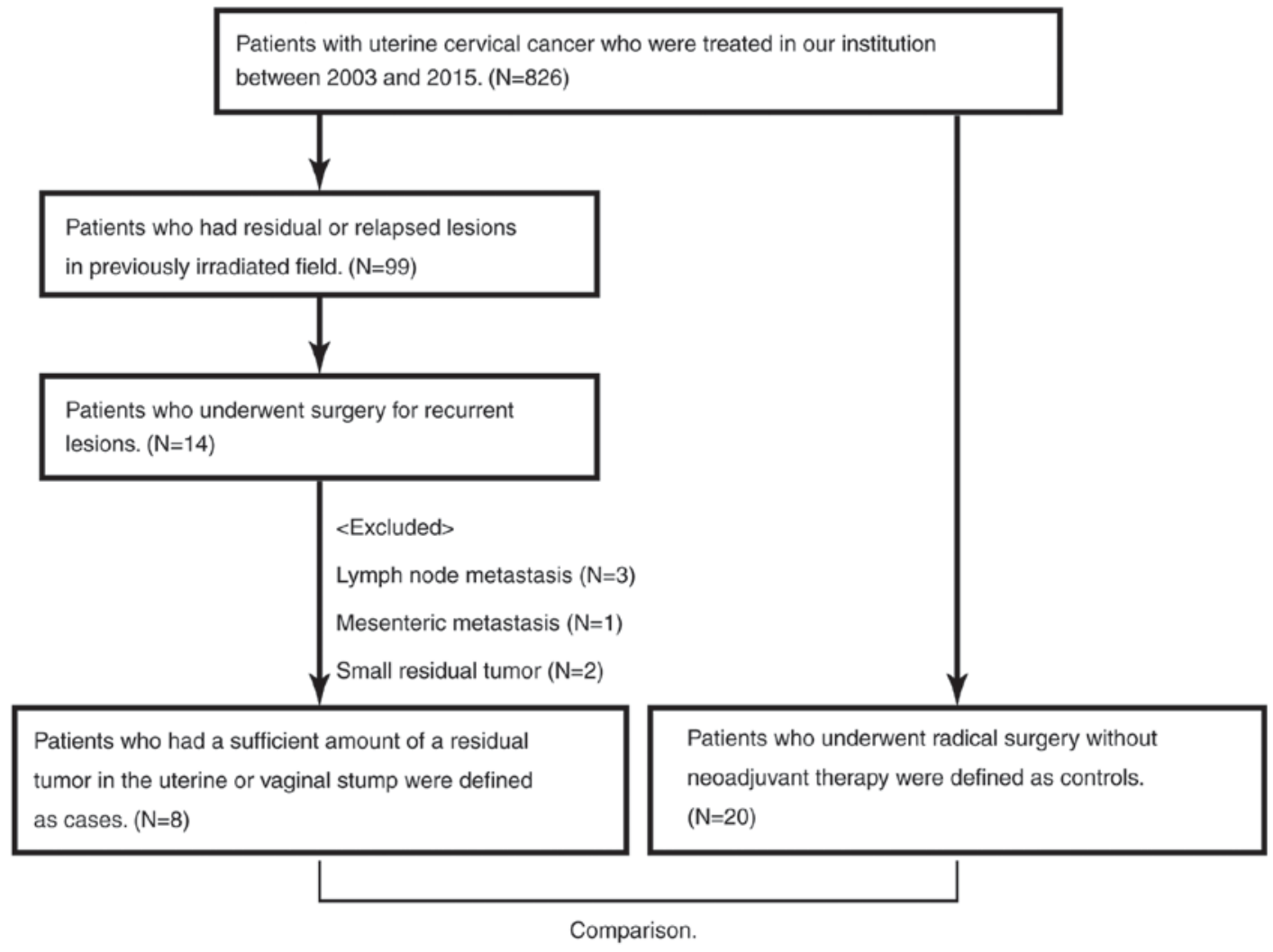

Figure 1. Flowchart of patient inclusion.

cells was weak (Spearman's correlation coefficient: 0.258 ; $\mathrm{P}=0.185$ ) (Fig. 3C).
The expression of HIF-1 $\alpha$ in carcinoma cells was also significantly stronger in the PRRCC group than control group, 
Table II. The evaluation of immunohistochemistry.

A, Cancer area

\begin{tabular}{lcccc}
\hline & \multicolumn{4}{c}{ Intensity } \\
\cline { 2 - 5 } Cancer area & Negative & Weak & Medium & Strong \\
\hline Focal & 0 & 1 & 1 & 2 \\
Sporadic & 0 & 1 & 2 & 3 \\
Diffuse & 0 & 2 & 2 & 3 \\
\hline
\end{tabular}

B, Stroma area

\begin{tabular}{lcccc}
\hline & \multicolumn{4}{c}{ Intensity } \\
\cline { 2 - 5 } Stroma area & Negative & Weak & Medium & Strong \\
\hline Focal & 0 & 1 & 1 & \\
Sporadic & 0 & 1 & 2 & \\
Diffuse & 0 & 2 & 3 & \\
\hline
\end{tabular}

although that in stroma cells was weak and showed no significant difference $(\mathrm{P}=0.343)$ (Fig. $2 \mathrm{C}$ and Table VC). Moreover, the expression of HIF-1 $\alpha$ was significantly correlated with that of VEGF-A in carcinoma cells, but not in stroma cells [Spearman's correlation coefficient: 0.797; $\mathrm{P}<0.0001$ (carcinoma); $\mathrm{P}=0.343$ (stroma)] (Fig. 3D).

\section{Discussion}

VEGF is an important factor for tumor angiogenesis, and there have been a number of reports evaluating the VEGF expression of primary surgery specimens or that of serum in uterine cervical cancer (12-16). However, to the best of our knowledge, there have been no reports concerning VEGF expression in PRRCC. In this study, we investigated the expression of VEGF and related molecules using tumor samples from patients with PRRCC. The expressions of both VEGF-A and VEGFR-1 were significantly higher in PRRCC sections than in controls. These results led us to hypothesize two possible mechanisms: 'natural selection' and 'evolution'. Intra-tumor genetic heterogeneity is also known in cervical cancer, and subpopulations of each tumor showed differential responses to chemoradiotherapy (20). In addition, patients with high VEGF expression in cancer tissue or serum were associated with a poor response to radiotherapy and poor survival (12-15). Therefore, the 'natural selection' hypothesis suggests that subpopulations of cervical cancer with high VEGF expression survive through chemoradiation, and then these selected subpopulations develop, leading to recurrence. On the other hand, in addition to its therapeutic effects, ionizing radiation is known to promote the malignant behaviors of surviving cancer cells. Radiation induced HIF- $1 \alpha$ and VEGF, and those factors were related to radioresistance $(21,22)$. Therefore, the 'evolution' hypothesis suggests that some cervical cancer cells are evolutionarily induced to acquire VEGF expression by radiation while most of them are killed, and then cancer with acquired
Table III. Patients' characteristics.

\begin{tabular}{|c|c|c|c|c|}
\hline \multirow[b]{2}{*}{ Characteristic } & \multicolumn{2}{|c|}{ Cases $(\mathrm{N}=8)$} & \multirow{2}{*}{$\begin{array}{l}\text { Controls } \\
(\mathrm{N}=20)\end{array}$} & \multirow[b]{2}{*}{ P-value } \\
\hline & $\# 1$ & $\# 2$ & & \\
\hline Age & & & & 0.321 \\
\hline Median & 43 & 44 & 39 & \\
\hline (Range) & $(34-73)$ & $(34-74)$ & $(20-68)$ & \\
\hline Stage & & & & 0.795 \\
\hline CIS & 1 & & 0 & \\
\hline Stage I & 3 & & 10 & \\
\hline Stage II & 4 & & 10 & \\
\hline Tumor size & & & & 0.100 \\
\hline$<4 \mathrm{~cm}$ & 5 & 8 & 18 & \\
\hline$\geq 4 \mathrm{~cm}$ & 3 & 0 & 2 & \\
\hline LVSI & & & & 0.591 \\
\hline Yes & & 6 & 15 & \\
\hline No & & 1 & 5 & \\
\hline Unknown & & 1 & 0 & \\
\hline Nodal metastasis & & & & 0.576 \\
\hline Yes & 3 & 1 & 10 & \\
\hline No & 5 & 0 & 10 & \\
\hline Unknown & & 7 & & \\
\hline \multicolumn{5}{|l|}{ Serum SCC level } \\
\hline$<2.0 \mathrm{ng} / \mathrm{ml}$ & 3 & 4 & 8 & 0.660 \\
\hline$\geq 2.0 \mathrm{ng} / \mathrm{ml}$ & 4 & 4 & 12 & \\
\hline Unknown & 1 & & & \\
\hline Previous treatment & & & & $<0.0001$ \\
\hline CCRT & & 5 & 0 & \\
\hline RT & & 3 & 0 & \\
\hline
\end{tabular}

\#1, at diagnosis of uterine cervical cancer; \#2, at recurrent diagnosis; CIS, carcinoma in situ; LVSI, lymphovascular space invasion; SCC, squamous cell carcinoma; CCRT, concurrent chemoradiotherapy; RT, radiotherapy.

resistance develops, leading to recurrence. Regardless of the two independent hypotheses, if VEGF and its receptor are upregulated in PRRCC, VEGF-targeting therapy is expected as an effective therapeutic strategy for this tumor.

Hypoxia is an important cancer microenvironment, and most solid human cancers including cervical cancer are known to induce such an environment (23). It is possible that PRRCC tissue is exposed to hypoxic conditions by tissue fibrosis after radiotherapy. HIF-1 $\alpha$ has been reported to mediate essential homeostatic responses by activating the transcription of multiple genes including VEGF (24). Indeed, in the present study, we showed that the expression of HIF-1 $\alpha$ in carcinoma cells was also significantly higher in the PRRCC than control group consistent with our current findings. According to earlier studies, hypoxic conditions enhanced the radiation resistance dependent on HIF-1 $\alpha$ by elevating the expression of VEGF and inhibiting the expression of p53 (25). In addition, high-level expression of HIF- $1 \alpha$ is associated with treatment-resistance, and, conversely, the inhibition of HIF-1 $\alpha$ transactivation 
Table IV. Characteristics and oncologic outcomes of patients with recurrence.

\begin{tabular}{|c|c|c|c|c|c|c|c|}
\hline No. & $\mathrm{Age}^{\mathrm{a}}$ & TNM & $\mathrm{Age}^{\mathrm{b}}$ & $\begin{array}{c}\text { PFS } \\
\text { (months) }\end{array}$ & Previous treatments & $\begin{array}{c}\text { OS } \\
\text { (months) }\end{array}$ & Outcome \\
\hline 1 & 34 & cT1b2N1M0 & 34 & 3 & CCRT (PFx4 kur + WP 56.4 Gy, RALS 15 Gy) & 20 & DOD \\
\hline 2 & 35 & pTisNOM0 & 38 & 16 & VTH $\rightarrow$ CCRT (PF x 5 kur + WP 50.4 Gy, RALS 13 Gy) & 47 & NED \\
\hline 3 & 37 & $\mathrm{cT} 2 \mathrm{bNOM} 0$ & 40 & 4 & $\begin{array}{l}\text { CCRT }(\text { PFx } 5 \text { kur + WP } 50.4 \text { Gy, RALS } 16 \text { Gy) } \rightarrow \text { TC } \\
\text { x6 kur } \rightarrow \text { CPT-11 x3 kur }\end{array}$ & 44 & NED \\
\hline 4 & 42 & cT1b2N0M0 & 43 & 7 & CCRT (PFx2 kur + WP 50.4 Gy, RALS 24 Gy) & 13 & DOD \\
\hline 5 & 44 & $\mathrm{cT} 2 \mathrm{bN} 1 \mathrm{M} 0$ & 45 & 16 & $\begin{array}{l}\text { CCRT (PFx1 kur, CBDCA x1 kur, NDP x3 kur + } \\
\text { WP } 50.4 \text { Gy, RALS } 18 \text { Gy) }\end{array}$ & 41 & NED \\
\hline 6 & 53 & cT1bNOM0 & 54 & 5 & RT (WP 50.4 Gy, RALS 24 Gy) $\rightarrow$ PF x2 kur $\rightarrow$ TP x4 kur & 24 & DOD \\
\hline 7 & 73 & cT2bNOM0 & 74 & 5 & RT (WP 50.4 Gy, RALS 13 Gy) & 35 & DOD \\
\hline 8 & 73 & cT2aN0M0 & 74 & 10 & RT (WP 50.4 Gy, RALS 9 Gy) & 14 & NED \\
\hline
\end{tabular}

${ }^{\mathrm{a} A t}$ diagnosis of uterine cervical cancer, ${ }^{\mathrm{b}}$ At recurrent diagnosis. PFS, progression free survival; OS, overall survival; VTH, vaginal total hysterectomy; CCRT, concurrent chemoradiotherapy; RT, radiotherapy; WP, whole pelvis; RALS, remote after loading system; PF, cisplatin and 5-FU; TC, paclitaxel and carboplatin; CPT-11, irinotecan; CBDCA, carboplatin; NDP, nedaplatin; TP, paclitaxel and cisplatin; DOD, died of disease; NED, no evidence of disease.

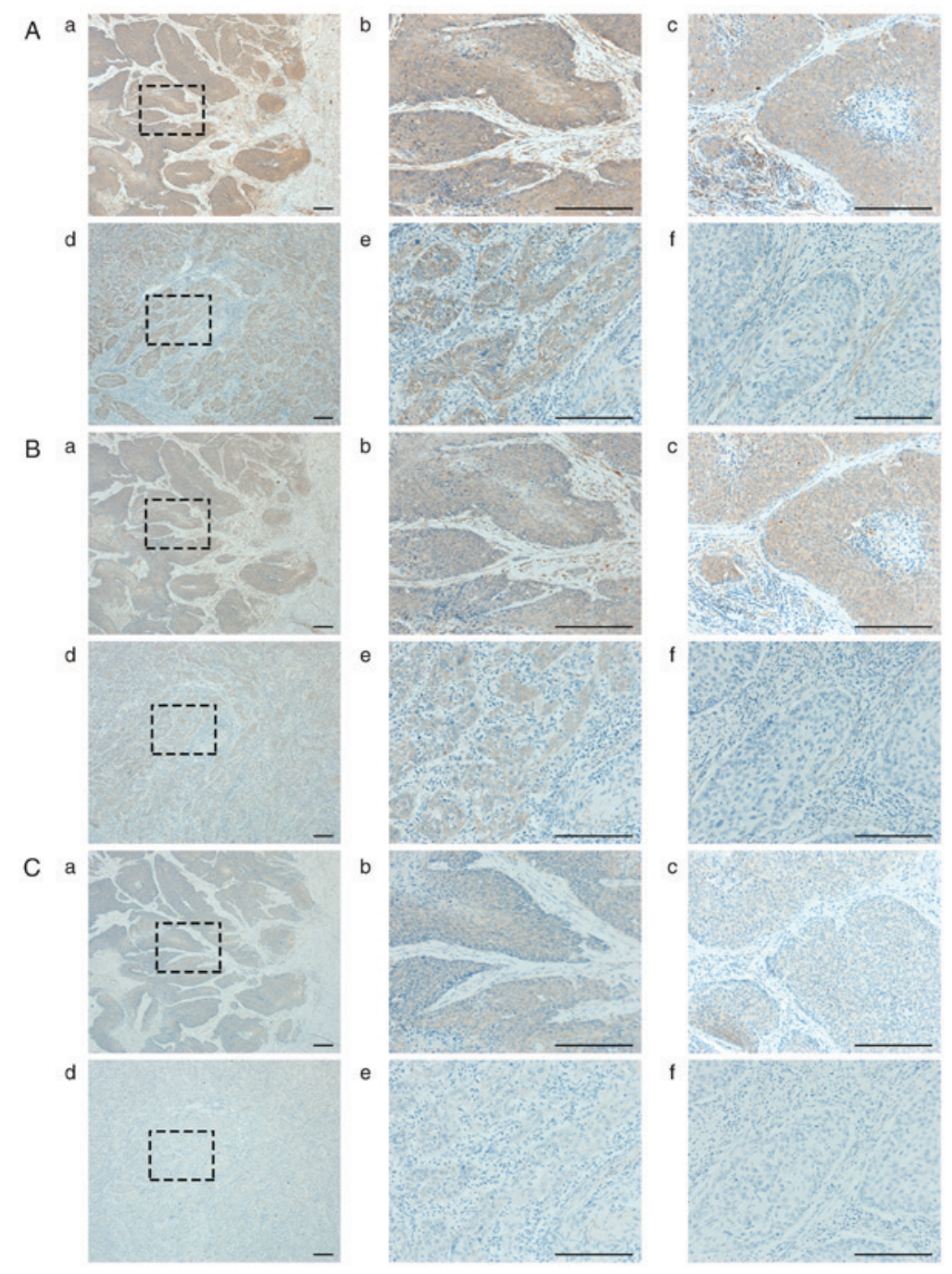

Figure 2. Representative images of immunohistochemistry. (A, B, and C) Representative images of VEGF-A, VEGFR-1, and HIF-1 $\alpha$, respectively. a and b show those of case 3, c shows that of case 2, $d$ and e show those of control 1, and f shows that of control 2. All scale bars, $200 \mu \mathrm{m}$. A-b shows strong expression in cancer (cancer-3), A-c: cancer-3, A-e: cancer-2, and A-f: cancer-0. A-b shows strong expression in stroma (stroma-3), A-c: stroma-3, A-e: stroma-0, and A-f: stroma-1. B-b: cancer-3, B-c: cancer-3, B-e: cancer-2, and A-f: cancer-0. B-b: stroma-3, B-c: stroma-2, B-e: stroma-0, and A-f: stroma-1. C-b: cancer-2, C-c: cancer-2, C-e: cancer-1, and A-f: cancer-0. C-b: stroma-1, C-c: stroma-1, C-e: stroma-0, and A-f: stroma-0. 
Table V. The expressions of VEGF-A, VEGFR-1, and HIF-1 $\alpha$.

A, VEGF-A expression

\begin{tabular}{lccccc}
\hline VEGF-A & Negative & Weak & Moderate & Strong & P-value \\
\hline Cancer & & & & & \\
Cases & & & 2 & 6 & 0.0003 \\
Controls & 6 & 8 & 5 & 1 & \\
Stroma & & & & & \\
Cases & 2 & 1 & & 5 & 0.0014 \\
Controls & 16 & 4 & & & \\
\end{tabular}

\section{B, VEGFR-1 expression}

VEGFR-1 Negative Weak Moderate Strong P-value

\begin{tabular}{lccccc}
\hline Cancer & & & & & \\
Cases & & & 2 & 6 & 0.0003 \\
Controls & 4 & 9 & 6 & 1 & \\
Stroma & & & & & \\
Cases & & & 5 & 3 & $<0.0001$ \\
Controls & 13 & 7 & & & \\
\hline
\end{tabular}

C, HIF-1 $\alpha$ expression

\begin{tabular}{lccccc}
\hline HIF-1 $\alpha$ & Negative & Weak & Moderate & Strong & P-value \\
\hline Cancer & & & & \\
Cases & 1 & 3 & 4 & 0.0001 \\
Controls & 18 & 1 & 1 & \\
Stroma & & & & 0.343 \\
Cases & 4 & 4 & & \\
Controls & 14 & 6 & & \\
\hline
\end{tabular}

enhances radiotherapy responses $(23,26)$. Burri et al reported that multivariate analyses revealed HIF-1 $\alpha$ expression to be an independent factor for overall survival based on an immunohistochemical analysis of 78 patients with uterine cervix carcinoma treated with external beam radiotherapy (27). This evidence prompted us to hypothesize that HIF-1 $\alpha$ plays a crucial role in VEGF upregulation and the treatment refractoriness of PRRCC.

In the current study, high-level expressions of VEGF-A and VEGFR-1 were observed in the stroma as well as in carcinoma cells. Cancer-associated fibroblasts (CAFs) are major components of the tumor stroma and involved in tumor progression. A previous report demonstrated the effects to protect against radiation of CAF-cancer cell crosstalk through multiple growth factors including VEGF in vitro (28). In order to inhibit VEGF-VEGFR interactions between carcinoma and stroma cells, VEGF inhibitors such as bevacizumab have been widely used. In cervical cancer, VEGF inhibitors also showed clinical benefits for patients with advanced, persistent, or recurrent lesions $(18,19,29)$. Especially, according to Tewari's sub-group analysis, bevacizumab was more favorable
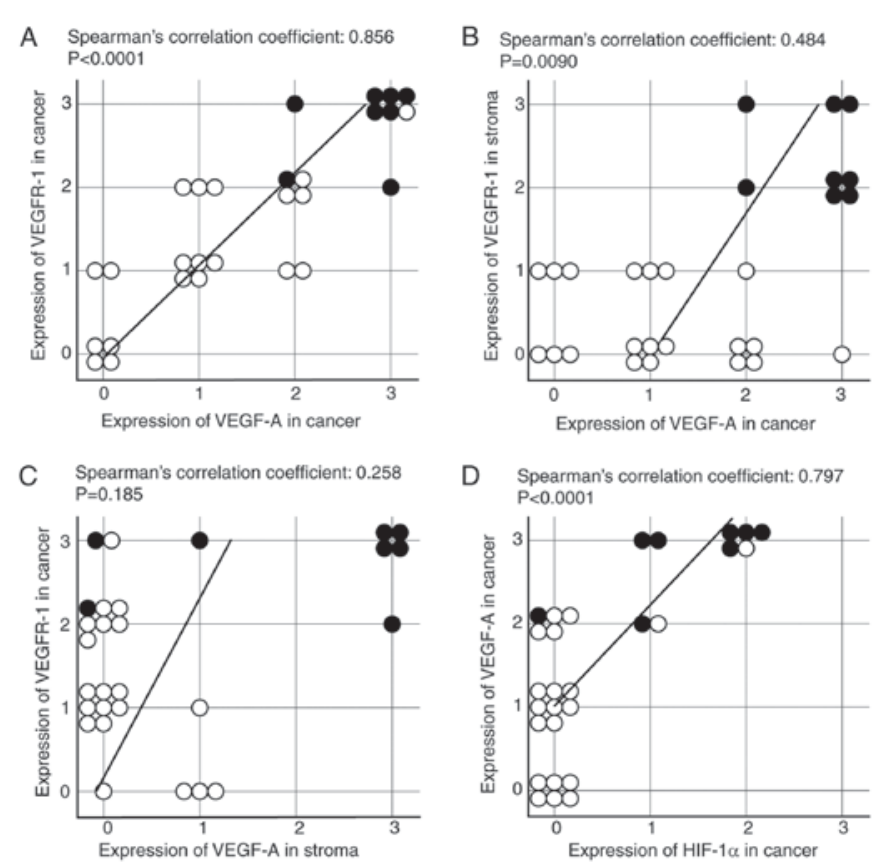

D Spearman's correlation coefficient: 0.797

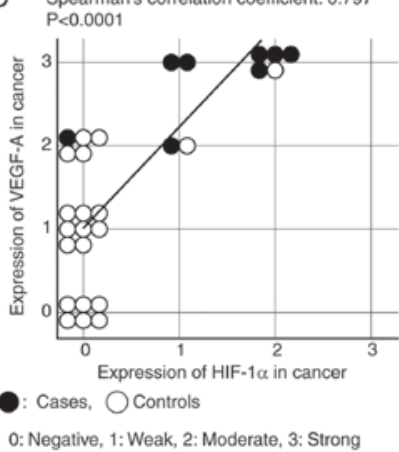

0: Negative, 1: Weak, 2: Moderate, 3: Strong

Figure 3. The correlations among the expressions of VEGF-A, VEGFR-1, and HIF-1 $\alpha$. (A) The correlation between the expression of VEGF-A in cancer and that of VEGFR-1 in cancer. (B) The correlation between the expression of VEGF-A in cancer and that of VEGFR-1 in the stroma. (C) The correlation between the expression of VEGF-A in the stroma and that of VEGFR-1 in cancer. (D) The correlation between the expression of HIF-1 $\alpha$ in cancer and that of VEGF-A in cancer.

in patients with recurrent or persistent lesions than those with advanced lesions, and also in those who previously received chemoradiotherapy (18). These results suggest that recurrent or persistent cancer after chemoradiotherapy expressed VEGF-A and VEGFR-1 more strongly than advanced cancer, being consistent with our results.

The main limitation of this study was the fact that only eight patients with PRRCC were available despite the enrollment of over 800 patients with cervical cancer. This limited patient number is consistent with the actual clinical situation whereby the selection of surgical treatment for PRRCC is extremely rare. Second, we did not evaluate the association between the VEGF expression and efficacy of VEGF inhibitors in patients with PRRCC. Moreover, we could not directly compare the immunohistochemical expressions between preand post-treatment sample sets in the same patient. Actually, it was difficult to obtain enough specimens from patients with primary CCRT before treatment. As a result, we used specimens of primary surgery as a control. An additional large-scale study to confirm our current findings is desirable by accumulating more patients with PRRCC from multiple institutions. Therefore, in the present study, we could not verify the direct effect of the radiation-induced expression of VEGF in tumor tissues of patients with PRRCC. We would like to verify the radiation-induced upregulation of VEGF expression effects in vitro and using an animal model in a future study.

In conclusion, the expressions of VEGF-A and VEGFR-1 were significantly upregulated in PRRCC. These results are important and valuable because there has been no evidence 
of VEGF expression in PRRCC. For further evaluation, a large-scale study of VEGF in advanced, residual, and recurrent cervical cancer is desired and the efficacy of VEGF inhibitors must be investigated. The prognoses of these patients are expected to improve in the future. We believe that our results will help clarify the efficacy of bevacizumab.

\section{Acknowledgements}

We sincerely thank members of the Deptartment of OBGYN, Nagoya University for collaborating in data collection.

\section{References}

1. Siegel RL, Miller KD and Jemal A: Cancer statistics, 2016. CA Cancer J Clin 66: 7-30, 2016

2. Morris M, Eifel PJ, Lu J, Grigsby PW, Levenback C, Stevens RE, Rotman M, Gershenson DM and Mutch DG: Pelvic radiation with concurrent chemotherapy compared with pelvic and para-aortic radiation for high-risk cervical cancer. N Engl J Med 340: $1137-1143,1999$.

3. Green JA, Kirwan JM, Tierney JF, Symonds P, Fresco L, Collingwood M and Williams CJ: Survival and recurrence after concomitant chemotherapy and radiotherapy for cancer of the uterine cervix: A systematic review and meta-analysis. Lancet 358: 781-786, 2001

4. Benedet JL, Odicino F, Maisonneuve P, Beller U, Creasman WT, Heintz AP, Ngan HY and Pecorelli S: Carcinoma of the cervix uteri. Int J Gynaecol Obstet 83 (Suppl 1): S41-S78, 2003.

5. Mabuchi S, Isohashi F, Yoshioka Y, Temma K, Takeda T, Yamamoto T, Enomoto T, Morishige K, Inoue T and Kimura T: Prognostic factors for survival in patients with recurrent cervical cancer previously treated with radiotherapy. Int J Gynecol Cancer 20: 834-840, 2010.

6. Waggoner SE: Cervical cancer. Lancet 361: 2217-2225, 2003

7. Legge F, Chiantera V, Macchia G, Fagotti A, Fanfani F, Ercoli A, Gallotta V, Morganti AG, Valentini V, Scambia G and Ferrandina G: Clinical outcome of recurrent locally advanced cervical cancer (LACC) submitted to primary multimodality therapies. Gynecol Oncol 138: 83-88, 2015.

8. Kosmas C, Mylonakis N, Tsakonas G, Vorgias G, Karvounis N, Tsavaris N, Daladimos T, Kalinoglou N, Malamos N, Akrivos T and Karabelis A: Evaluation of the paclitaxel-ifosfamide-cisplatin (TIP) combination in relapsed and/or metastatic cervical cancer. Br J Cancer 101: 1059-1065, 2009.

9. Benjapibal M, Thirapakawong C, Leelaphatanadit C, Therasakvichya S and Inthasorn P: A pilot phase II study of capecitabine plus cisplatin in the treatment of recurrent carcinoma of the uterine cervix. Oncology 72: 33-38, 2007.

10. Borcoman E and Le Tourneau C: Pembrolizumab in cervical cancer: Latest evidence and clinical usefulness. Ther Adv Med Oncol 9: 431-439, 2017.

11. Dallas NA, Fan F, Gray MJ, Van Buren G II, Lim SJ, Xia L and Ellis LM: Functional significance of vascular endothelial growth factor receptors on gastrointestinal cancer cells. Cancer Metastasis Rev 26: 433-441, 2007.

12. Loncaster JA, Cooper RA, Logue JP, Davidson SE, Hunter RD and West CM: Vascular endothelial growth factor (VEGF) expression is a prognostic factor for radiotherapy outcome in advanced carcinoma of the cervix. Br J Cancer 83: 620-625, 2000.

13. Lee IJ, Park KR, Lee KK, Song JS, Lee KG, Lee JY, Cha DS, Choi HI, Kim DH and Deung YK: Prognostic value of vascular endothelial growth factor in Stage IB carcinoma of the uterine cervix. Int J Radiat Oncol Biol Phys 54: 768-779, 2002.

14. Bachtiary B, Selzer E, Knocke TH, Pötter R and Obermair A Serum VEGF levels in patients undergoing primary radiotherapy for cervical cancer: Impact on progression-free survival. Cancer Lett 179: 197-203, 2002.
15. Cheng WF, Chen CA, Lee CN, Wei LH, Hsieh FJ and Hsieh CY: Vascular endothelial growth factor and prognosis of cervical carcinoma. Obstet Gynecol 96: 721-726, 2000.

16. Iwasaki K, Yabushita H, Ueno T and Wakatsuki A: Role of hypoxia-inducible factor-1 $\alpha$, carbonic anhydrase-IX, glucose transporter-1 and vascular endothelial growth factor associated with lymph node metastasis and recurrence in patients with locally advanced cervical cancer. Oncol Lett 10: 1970-1978, 2015.

17. Zhang J, Liu J, Zhu C, He J, Chen J, Liang Y, Yang F, Wu X and Ma X: Prognostic role of vascular endothelial growth factor in cervical cancer: A meta-analysis. Oncotarget 8: 24797-24803, 2017.

18. Tewari KS, Sill MW, Long HJ III, Penson RT, Huang H, Ramondetta LM, Landrum LM, Oaknin A, Reid TJ, Leitao MM, et al: Improved survival with bevacizumab in advanced cervical cancer. N Engl J Med 370: 734-743, 2014.

19. Schefter T, Winter K, Kwon JS, Stuhr K, Balaraj K, Yaremko BP, Small W Jr, Sause W and Gaffney D; Radiation Therapy Oncology Group (RTOG): RTOG 0417: efficacy of bevacizumab in combination with definitive radiation therapy and cisplatin chemotherapy in untreated patients with locally advanced cervical carcinoma. Int J Radiat Oncol Biol Phys 88: 101-105, 2014.

20. Cooke SL, Temple J, Macarthur S, Zahra MA, Tan LT, Crawford RA, Ng CK, Jimenez-Linan M, Sala E and Brenton JD: Intra-tumour genetic heterogeneity and poor chemoradiotherapy response in cervical cancer. Br J Cancer 104: 361-368, 2011.

21. Gorski DH, Beckett MA, Jaskowiak NT, Calvin DP, Mauceri HJ, Salloum RM, Seetharam S, Koons A, Hari DM, Kufe DW and Weichselbaum RR: Blockage of the vascular endothelial growth factor stress response increases the antitumor effects of ionizing radiation. Cancer Res 59: 3374-3378, 1999.

22. Moeller BJ, Cao Y, Li CY and Dewhirst MW: Radiation activates HIF-1 to regulate vascular radiosensitivity in tumors: Role of reoxygenation, free radicals, and stress granules. Cancer Cell 5: 429-441, 2004

23. Rey S, Schito L, Koritzinsky M and Wouters BG: Molecular targeting of hypoxia in radiotherapy. Adv Drug Deliv Rev 15: 45-62, 2017.

24. Jiang BH, Agani F, Passaniti A and Semenza GL: V-SRC induces expression of hypoxia-inducible factor 1 (HIF-1) and transcription of genes encoding vascular endothelial growth factor and enolase 1: Involvement of HIF-1 in tumor progression. Cancer Res 57: 5328-5335, 1997.

25. Fu Z, Chen D, Cheng H and Wang F: Hypoxia-inducible factor- $1 \alpha$ protects cervical carcinoma cells from apoptosis induced by radiation via modulation of vascular endothelial growth factor and p53 under hypoxia. Med Sci Monit 21: 318-325, 2015.

26. Dewhirst MW, Cao Y and Moeller B: Cycling hypoxia and free radicals regulate angiogenesis and radiotherapy response. Nat Rev Cancer 8: 425-437, 2008.

27. Burri P, Djonov V, Aebersold DM, Lindel K, Studer U, Altermatt HJ, Mazzucchelli L, Greiner RH and Gruber G: Significant correlation of hypoxia-inducible factor-1alpha with treatment outcome in cervical cancer treated with radical radiotherapy. Int J Radiat Oncol Biol Phys 56: 494-501, 2003.

28. Chu TY, Yang JT, Huang TH and Liu HW: Crosstalk with cancer-associated fibroblasts increases the growth and radiation survival of cervical cancer cells. Radiat Res 181: 540-547, 2014.

29. Symonds RP, Gourley C, Davidson S, Carty K, McCartney E, Rai D, Banerjee S, Jackson D, Lord R, McCormack M, et al: Cediranib combined with carboplatin and paclitaxel in patients with metastatic or recurrent cervical cancer (CIRCCa): A randomised, double-blind, placebo-controlled phase 2 trial. Lancet Oncol 16: 1515-1524, 2015. 